\title{
PROJECTIVE IDEALS IN RINGS OF DIMENSION ONE
}

\author{
J. E. CARRIG AND W. V. VASCONCELOS ${ }^{1}$
}

\begin{abstract}
In this paper we discuss a construction that shows the projective ideals of a one-dimensional ring as radically dense in the set of all faithful ideals. Its applications are to questions of existence of a classical ring of quotients, to counting equations, and to a trade-in between Noetherianess and low homological dimension.
\end{abstract}

0. Introduction. Let $R$ be a commutative ring of Krull dimension 1 . We put a strong arithmetical limitation on $R$ by assuming that the Krull dimension of $R[T]$ be 2 - a condition that, for integral domains, implies that the integral closure of $R$ is a Prüfer ring [10]. This restriction will hold unless otherwise specified, as in the applications.

The main result of this note is the following. Throughout $\vee I$ denotes the radical of the ideal $I$.

THEOREM. Let I be a finitely generated faithful ideal of a commutative ring $R$. If the Krull dimension of $R[T]$ is two, there exists a projective ideal $J$ with $V_{I}=V_{J}$.

The applications of this theorem are somewhat restricted to those cases where the Picard group of $R$ is adequately circumscribed. This will be specially true for questions on determining the classical ring of quotients of non-Noetherian rings. Its usefulness extends to higher dimensional rings though, as the proof that a Kneser bound (whether for a given $d$-dimensional Noetherian ring a radical ideal is the radical of a $d$-generated ideal) is also valid for affine rings over the integers or a finite field.

1. Proof. It consists of a number of relatively independent steps. We begin with the critical one.

(1) Assume that for some integer $n$ the ideal $I^{n}$ is projective over its endomorphism ring $S$, that is, $I^{n}$ is stable in the terminology of [9]. Change notation and let $I$ stand for $I^{n}$. Note that $S$ is commutative and integral over $R$ and thus has Krull dimension 1 also. Since $I$ can be viewed as an ideal of $S$, we have that $I / I^{2}$ is a cyclic $S$-module [9]. Thus we may write $I=$ $\left(I^{2}, S d\right)$, for some $d \in I$. Note that $I^{2}=\left(I^{4}, d I^{2}, S d^{2}\right)=\left(I^{4}, I d\right)$.

Received by the editors November 11, 1977.

AMS (MOS) subject classifications (1970). Primary 13C10; Secondary 13 F05.

Key words and phrases. Projective ideal, Picard group, affine algebra, ring of quotients, stable coherence.

${ }^{1}$ The second author was partially supported by a National Science Foundation grant. 
Put $J=\left(I^{2}, d\right)$. We claim that $J$ is a projective ideal of $R$. It will suffice to show that $J / J^{2}$ is a cyclic $R$-module. Because of the identity on $I^{2}$ above, we have $J=\left(I^{4}, d\right)$. Thus $J / J^{2}$ is generated by the class of $d$.

(2) We shall make use of two remarks on the endomorphism ring $S$ of a finitely generated faithful ideal $I$. Recall that $S$ is a commutative ring.

(a) If $R$ is local then $S$ is a semilocal ring. This is generally true of any finitely generated $R$-module $E$; that is, $\operatorname{Hom}_{R}(E, E)$ has only finitely many two-sided maximal ideals. It follows, say, from the fact that epimorphisms of finitely generated modules are isomorphisms [12].

(b) For an indeterminate $T$ the canonical mapping

$$
\operatorname{Hom}_{R}(I, I) \otimes_{R} R[T] \rightarrow \operatorname{Hom}_{R}[T](I[T], I[T])
$$

is an isomorphism. One way to see this is to add still another indeterminate, 'exchange' indeterminates, use that $I[T]$ contains regular elements and apply flat descent. If $R$ is local with maximal ideal $\mathbf{m}$, the complement $U$ of $\mathbf{m}[T]$ consists of regular elements. It follows easily now that formation of the endomorphism ring of $I$ commutes with tensorization by $R[T]_{U}$. In this manner we introduce regular elements in $I$ and make the residue field of the ring infinite.

(3) If $R$ is a local ring and $I$ is stable, $I=S d$ since $S$ is semilocal. Thus $I^{2}=I d$. Conversely, if such equation holds, as $d$ is a regular element $I d^{-1}$ is a ring contained in $S$, and $I$ is $I d^{-1}$-projective. This equation is a more convenient way to test stability.

From the compactness of Spec $R$ it follows easily that if for each prime ideal $P$ some power of $I_{P}$ is stable, then some power of $I$ is stable. Since $I$ may be assumed to contain regular elements and for such ideals formation of the endomorphism ring commutes with localization, we take $R$ local.

(4) Assume that $I$ contains regular elements and that $B I$ is $B$-projective, where $B$ is the integral closure of $R$. We claim that some power of $I$ is stable. First, we may assume that $A I$ is $A$-projective, where $A$ is a finitely generated $R$-subring of $B$. Then $A I=A d$ with $d \in I$ since the residue field of $R$ may be taken to be infinite. It follows easily that $\left(I d^{-1}\right)^{n}=\left(I d^{-1}\right)^{n+1}$ for some large $n$. Thus $I^{n}$ is stable.

(5) We now make use of the hypothesis $\operatorname{dim} R[T]=2$ to show that some power of $I$ is stable. By (4) this is true modulo each prime of $R$ since $R / \mathbf{p}$ has a Prüfer ring for its integral closure. Suppose first that $R$ is reduced. According to [3] to show some power of $I$ stable it suffices to show that the number of generators of $I$ is bounded. Let $a_{1}, \ldots, a_{r}$ be a generating set for $I$ (could be taken minimal). Consider the following ring homomorphism

$$
\phi: R\left[T_{1}, \ldots, T_{r}\right] \rightarrow R[T]
$$

$\phi\left(T_{i}\right)=a_{i} T . \phi$ is a graded map; let $H$ be its kernel. We claim that the ideal of $R$ generated by the coefficients of polynomials in $H$ is $R$ itself. That this will suffice to show that the powers of $I$ have bounded number of generators can be viewed as in [3]. Let then $P$ be a prime ideal of $R\left[T_{1}, \ldots, T_{r}\right]$ containing 
$H$; we shall show that $P$ contains a polynomial of content $R$. Let $\mathrm{p}$ be a minimal prime of $R$ contained in $P$. Reduce the homomorphism above modulo p. Now by (4) the ideal $I^{*}=I+\mathbf{p} / \mathbf{p}$ has a power that is stable. This translates into: There exists a homogeneous polynomial $f\left(T_{1}, \ldots, T_{r}\right)$ of content $R$ such that $f\left(a_{1} T, \ldots, a_{r} T\right) \in \mathbf{p}[T]$. The conclusion now follows.

We have shown that modulo the nilradical of $R$ some power $J$ of $I$ is stable. That is, we have an equation $J^{2}=J d+L$, where $L$ is a finitely generated ideal contained in the radical. Raising this equation to an adequate power we get $J^{m}=d J^{m-1}$ and some power of $J$ is stable.

The proof of the theorem is now complete. Note that for Noetherian rings most of this hassle is unnecessary.

2. Applications. We consider here some immediate consequences of the theorem. Let $R$ be a Noetherian ring of Krull dimension $d>0$. We say that $R$ admits the Kneser bound [7] if every radical ideal is the radical of a $d$-generated ideal. It is known that every polynomial ring admits the Kneser bound [4], [11].

(2.1) Corollary. Let $R$ be such that the Picard groups of the onedimensional homomorphic images of $R$ are torsion-e.g., affine algebras over $\mathbf{Z}$ or a finite field or even the algebraic closure of a finite field. Then $R$ admits the Kneser bound.

PROof. There are several approaches, the most natural being Kneser's. $R$ may be assumed reduced. Let $U$ be a multiplicative set of regular elements of $R$ such that $I_{U}=(f)_{U}, f \in I$. (For instance, let $U$ be the set of all regular elements.) Let $s \in U$ be such that $s I \subset(f)$. Consider the ideal $I^{*}=I+$ $(s) /(s)$ of $R^{*}=R /(s)$. If $f_{1}, \ldots, f_{d-1}$ are elements of $I$ such that

$$
\vee I^{*}=\sqrt{ }\left(f_{1}, \ldots, f_{d-1}\right)^{*},
$$

it follows easily that $\vee I=\sqrt{ }\left(f_{1}, \ldots, f_{d-1}, f\right)$. Thus it suffices to prove (2.1) when $R$ is one-dimensional. Let $0: I=L$; then $I+L$ is a faithful ideal. Let $J$ be as in the theorem. Since $\operatorname{Pic}(R)$ is a torsion group, some power of $J$ is principal, $J^{r}=(f)$. Thus $\sqrt{ }(f)=\sqrt{ }(I+L)$. If $f^{n}=g+h, g \in I, h \in L$, it follows that $\vee I=\sqrt{ }(g)$.

Another consequence is the following. Suppose $R$ is a two-dimensional affine algebra over $\mathbf{Z}$ or a finite field.

(2.2) COROLlaRY. If $R$ is a regular then $S K_{0}(R)$ is a torsion group.

Proof. This follows simply from the fact that $S K_{0}(R)$ is in this case generated by $[R / \mathbf{m}]$, for maximal ideals $\mathbf{m}$ of height two [8]. But $\mathbf{m}=\sqrt{ }(a, b)$ and $0=[R /(a, b)]=r[R / \mathbf{m}]$, where $r$ is the length of the module $R /(a, b)$.

From now on $R$ is not assumed Noetherian, but still Krull $\operatorname{dim} R[T]=2$.

(2.3) COROLLARY. If in the theorem I is contained in the Jacobson radical of $R$, then $\bigvee I=\bigvee(f)$. In particular I contains a regular element. 
Proof. In this case $J / J^{2}$ is principal and Nakayama lemma can be used.

For a local ring of dimension two a finitely generated faithful ideal does not necessarily contain regular elements [13].

Let $R$ be a semilocal ring with compact minimal prime spectrum. If $R$ is reduced we have a finitely generated ideal $I$ contained in the Jacobson radical of $R$ but not contained in any minimal prime. By (2.3) $I$ contains a regular element and thus $R$ has a von Neumann classical ring of quotients. Still the same conclusion is obtained when $R$ contains a field $K$ of cardinality higher than that of the minimal prime spectrum. Indeed, let $I$ be a finitely generated faithful ideal. We may assume $I$ projective. By [9] $I$ can be generated by two elements, say $I=(a, b)$. Consider the elements $\{a+r b, r \in K\}$. If they all belonged to minimal primes, for different $r$ 's we would have

$$
a+r_{1} b, a+r_{2} b \in P=\text { minimal prime. }
$$

But then $\left(r_{1}-r_{2}\right) b \in P$ and both $a$ and $b$ would lie in $P$, a contradiction.

Question. Do reduced rings with Krull $\operatorname{dim} R[T]=2$ and compact minimal spectrum always have a classical von Neumann ring of fractions?

3. Stable coherence. We use the theorem to study an aspect of stable coherence of a class of rings with low homological dimensions. Let $R$ be as before reduced with $\mathrm{Krull} \operatorname{dim} R[T]=2$. Assume also that $R$ is coherent and that its finitistic projective dimension-that is, the supremum of the dimensions of modules of finite projective dimension-is one.

\section{(3.1) COROLlaRY. Every nonminimal prime ideal is finitely generated.}

Proof. Let $P$ be a nonminimal prime and let $x \in P$ be such that $(x)_{P} \neq 0$. Let $L$ be the annihilator of $x$. Then $L$ is finitely generated and $I=(x, L)$ is a faithful ideal contained in $P$. Let $J$ be as in the theorem. Then $J \subset P$ and $R / J$ is a coherent ring of finitistic projective dimension 0 , by the change of rings theorem of [5]. Thus, $R / J$ is Artinian [1,2]; in particular $P$ is finitely generated.

There is a partial converse to the rings of (3.1). Moreover such rings are stably coherent in the sense of the following proposition.

(3.2) Proposition. Let $R$ be a reduced ring such that every nonminimal prime is finitely generated; then $R$ is coherent. Moreover, if $\mathrm{Krull} \operatorname{dim} R=1$, then $R\left[T_{1}, \ldots, T_{r}\right]$ is coherent.

Proof. (1) We must show that: (i) For $a \in R, 0: a$ is finitely generated; (ii) If $I, J$ are finitely generated, then $I \cap J$ is also finitely generated.

Let 0: $a=L$; then $(a)+L=(a) \oplus L$, since $R$ is reduced. As $(a)+L$ is not contained in any minimal prime, all primes containing it are finitely generated. Thus essentially by Cohen's theorem $(a)+L$ is finitely generated also.

Let $I, J$ be finitely generated ideals; as in the preceding, $I J+0: I J=I J \oplus$ $0: I J$ is finitely generated; since $0: I J=0:(I \cap J)$ and $I \cap J \oplus(0: I \cap J)$ $\supset I J \oplus 0: I J$, we conclude that $I \cap J$ is also finitely generated. 
(2) Assume now Krull $\operatorname{dim} R=1$. Note that $\operatorname{Krull} \operatorname{dim} R[T]=2$. Thus if $R(T)=R[T]_{U}$, where $U$ is the multiplicative set of polynomials of content $R$, then $\operatorname{dim} R(T)=1$ and its prime ideals are extensions of the primes of $R$. Thus $R(T)$ is coherent.

Suppose the statement on polynomial coherence is true for $n-1$ indeterminates. In particular $R\left(T_{1}\right)\left[T_{2}, \ldots, T_{n}\right]$ is coherent. Now we use a device of Gruson: For any index set $I$ the natural map

$$
\phi: R\left[T_{1}, \ldots, T_{n}\right]^{I} \rightarrow R\left(T_{1}\right)\left[T_{2}, \ldots, T_{n}\right]^{I}
$$

is $R\left[T_{2}, \ldots, T_{n}\right]$-pure. Since $R\left[T_{2}, \ldots, T_{n}\right]$ is coherent, $\operatorname{coker}(\phi)$ is $R\left[T_{2}, \ldots, T_{n}\right]$-flat. Thus by the syzygies' lemma [6], the flat dimension of coker $(\phi)$ over $R\left[T_{1}, \ldots, T_{n}\right]$ is at most one. Hence $R\left[T_{1}, \ldots, T_{n}\right]^{I}$ is flat and the proof is complete [2].

QUESTION. Is the dimension restriction necessary for the validity of (3.2)?

The rings of global dimension one, that is, hereditary rings, provide examples for which (3.2) applies. Whether examples of local rings exist is unknown to the authors.

\section{BIBLIOGRAPHY}

1. H. Bass, Finitistic dimension and a homological generalization of semi-primary rings, Trans. Amer. Math. Soc. 95 (1960), 466-488.

2. S. U. Chase, Direct product of modules, Trans. Amer. Math. Soc. 97 (1960), 457-473.

3. P. Eakin and A. Sathaye, Prestable ideals, J. Algebra 41 (1976), 439-454.

4. D. Eisenbud and E. G. Evans, Every algebraic set in $n$-space is the intersection of $n$ hypersurfaces, Invent. Math. 19 (1973), 107-112.

5. C. U. Jensen, Remarks on a change of rings theorem, Math. Z. 106 (1968), 395-401.

6. I. Kaplansky, Fields and rings, Univ. of Chicago Press, Chicago, 1969.

7. M. Kneser, Uber die Darstellung algebraischer Raumkurven als Durchschnitte von Flachen, Arch. Math. 11 (1960), 157-158.

8. M. P. Murthy and R. G. Swan, Vector bundles over affine surfaces, Invent. Math. 36 (1976), 125-165.

9. J. D. Sally and W. V. Vasconcelos, Stable rings, J. Pure Appl. Algebra 4 (1974), 319-336.

10. A. Seidenberg, A note on the dimension theory of rings, Pacific J. Math. 3 (1953), 505-512.

11. U. Storch, Bemerkung zu einem Satz von M. Kneser, Arch. Math. 23 (1972), 403-404.

12. W. V. Vasconcelos, On finitely generated flat modules, Trans. Amer. Math. Soc. 138 (1969), $505-512$.

13. __ Annihilators of modules with a finite free resolution, Proc. Amer. Math. Soc. 29 (1971), 440-442.

Department of Mathematics, George Mason University, Fairfax, Virginia 22030

Department of Mathematics, Rutgers University, New Brunswick, New Jersey 08903 